\title{
Code génétique et séléno-protéines
}

BRÈVES $\square$

Le sélénium est un oligo-élément jouant un rôle dans l'activité d'une classe d'enzymes, les séléno-enzymes, qui comprend notamment la glutathion peroxydase des eucaryotes et la formate déshydrogénase des procaryotes. Chez E. Coli, aussi bien que chez les mammifères, il a été montré que l'incorporation de sélénium dans les sélénopeptides, sous la forme de sélénocystéine, était un processus co-traductionnel et était commandée par un codon stop UGA dans la phase ouverte de lecture [1-3]. Afin d'élucider le mécanisme de cette utilisation d'un codon stop pour incorporer la sélénocystéine dans les protéines, une équipe franco-allemande a analysé différentes souches de E. Coli (obtenues par traitement avec un mutagène) déficientes en l'activité de la formate déshydrogénase [4]. Quatre types de mutants (c'est-à-dire quatre groupes de complémentation) ont été obtenus. L'un correspond à une altération du gène d'un ARN de transfert de la sérine, minoritaire dans la cellule. La sérine est chargée sur cet $\mathrm{ARNt^{ \text {ser } }}$ sous l'action de l'enzyme amino-acyl ARNtsynthétase habituelle. Du fait des caractéristiques structurales particulières de l'ARNt, le séryl-amino-acyl ARNt obtenu est alors le substrat spécifique d'enzymes de modification encore mal connues, transformant la sérine en sélénocystéine. L'anticodon de l'ARNt reconnaît alors le codon UGA (se comportant ainsi comme un suppresseur de codon stop UGA, aussi dénommé codon opale), incorporant la sélénocystéine dans la protéine au niveau de ce qui, ailleurs, aura la signification d'une fin de traduction.

Il existe, chez les eucaryotes, un ARNt suppresseur de codon opale dont la fonction est inconnue; il est chargé par un résidu sérine phosphorylé. Cet $A R \mathrm{Nt}^{\mathrm{o}-\mathrm{p}-\mathrm{ser}}$ suppresseur pourrait permettre l'incorporation directe de phosphosérine dans des protéines; il pourrait aussi être un intermédiaire réactionnel entre ARNt ${ }^{\text {ser }}$ et l'ARNt ${ }^{\text {se-cys }}[4,5]$.
La nature de l'information déterminant qu'un codon UGA sera utilisé pour incorporer de la sélénocystéine et non pour terminer une protéine reste absolument mystérieuse. Le contexte de séquence dans lequel s'insère cet UGA pourrait être en cause. Ces résultats soulèvent également des questions sur la signification originelle du codon opale UGA : était-il un codon stop ayant acquis, pour les besoins de l'évolution, une signification nouvelle? Était-il, au contraire, le codon sélénocystéine des organismes primitifs vivant en anaérobiose? La grande réactivité du sélénium à l'oxygène aurait, alors que se développait sur la terre la vie aérobie, éliminé les organismes comportant de nombreuses séléno-enzymes et rendu inutile, voire potentiellement dangereuse, l'utilisation générale d'UGA pour fabriquer des séléno-protéines. Le rôle de terminateur de traduction du codon opale serait alors un phénomène secondaire, son rôle maintenant exceptionnel de codon sélénocystéine étant un «fossile» de sa fonction initiale. Peut-être l'étude approfondie du code génétique de certaines archébactéries anaérobies permettra-t-elle de répondre à ces questions.

A.K

1. Zinoni F, Birkmann A, Stadtman TC, Böck A. Nucleotide sequence and expression of the selenocysteine containing polypeptide of formate dehydrogenase from Escherichia coli. Proc Natl Acad Sci USA $1986 ; 83$; 4650-4. 2. Chambers I, Frampton J, Goldfarb P, Affara N, McBain W, Harrison PA. The structure of the mouse glutathione peroxidase gene: the selenocysteine in the active site is encoded by the termination codon, TGA. EMBO J 1986; 5 : $1221-7$.

3. SukenagaY, IshidaK, Takeda T, Takagi K. cDNA sequence coding for human glutathione peroxydase. Nucleic Acids Res 1987 ; $15: 71-78$.

4. Leinfelder W, Zehelein E, Mandrand-Berthelot MA, Böck A. Gene for a novel tRNA species that accepts L-serine and co-translationnally inserts selenocysteine. Nature 1988; $331: 723-5$.

5. Söll D. Genetic code : enter a new aminoacid. Nature $1988 ; 331: 662-3$.
Droduction par génie génétique d'anticorps hybrides utilisables en thérapeutique humaine. L'immunothérapie passive peut être très intéressante en thérapeutique humaine pour détruire des cellules exprimant un antigène spécifique. Le sérum antilymphocytaire, d'origine animale, a ainsi été très utilisé à une certaine période. Une telle approche comporte cependant une très importante limitation: le développement d'anticorps dirigés contre l'immunoglobuline hétérospécifique. L'utilisation d'anticorps humains permettrait de surmonter cette difficulté, mais leur obtention est très difficile. La création par génie génétique de molécules hybrides est maintenant possible. Le laboratoire de Greg Winster (MRC, Cambridge, Grande Bretagne) vient ainsi de remodeler un gène d'immunoglobuline humaine en remplaçant les six parties hypervariables des régions variables de gènes codant pour des chaînes lourdes $\gamma$ et une chaîne légère $\kappa$ humaine par les domaines correspondants d'un gène de rat codant pour un anticorps monoclonal antilymphocytaire humain (dirigé contre l'antigène CAMPATH - 1 spécifique des cellules lympho-monocytaires). Après réintroduction dans des cellules lymphoïdes en culture, ce gène modifié code pour un anticorps ayant une forte spécificité antilymphocytaire humaine [1]. La même équipe, afin d'étendre la validité du modèle, est également parvenue à transférer à un anticorps humain une spécificité antilysozyme en remplaçant les régions hypervariables humaines par les régions correspondantes d'un anticorps antilysozyme de souris [2]. Les parties hypervariables remplacées ne semblent pas spécifiques d'espèce, si bien que les auteurs espèrent qu'une telle molécule n'entraînera pas une réponse anti-idiotypique trop importante.

[1. Rieckmann L, et al. Nature 1988; 332: 323-7.]

[2. Verhoeyen M, et al. Science 1988; 239: 1534-5.] 
Zinc et dyslexie. On a souvent cherché à corréler les troubles d'acquisition du langage et de la lecture à des anomalies de la concentration en certains métaux. Des dosages, cheveux, ont révélé une élévation du taux de certains métaux coınme le cuivre, le plomb, le cadmium. Des auteurs anglais se sont intéressés au zinc, dont l'importance pour le développement cérébral a été démontrée expérimentalement. Comme un déficit en zinc se reflète en une baisse du taux de ce métal dans la sueur, ils ont dosé par une méthode de spectrométrie d'absorption atomique le zinc et dix autres métaux dans la sueur et les cheveux chez 26 «paires » d'enfants de 6 à 14 ans, comprenant chacune un témoin et un dyslexique, appariés le mieux possible. La concentration moyenne du zinc dans la sueur des dyslexiques était de $66 \%$ de celle des témoins $(\mathrm{p}<0,0001) ; 25$ des 26 paires d'enfants montraient cette diminution. Une baisse plus discrète était observée pour le chrome, tandis que le cuivre était élevé. Dans les cheveux, si on retrouvait l'élévation du cuivre et la baisse du chrome, le zinc était normal. Il existe donc une association entre dyslexie et diminution de la concentration du zinc dans la sueur. Ce travail demande confirmation ; si celle-ci était obtenue, il pourrait conduire à des tentatives de supplémentation, et, pourquoi pas, à une amélioration des performances scolaires de certains enfants.

[Grant ECG, et al. Br Med J 1988; 296: 601-9.]

Oncogènes et facteurs de transcription. Il apparaît de plus en plus évident que les oncogènes de la famille myc et fos, dont les produits Myc et Fos sont des protéines nucléaires, agissent en modifiant la transcription d'autres gènes. La même chose est vraie des oncogènes viraux de type $E_{1} A$ d'adénovirus. Nous avons rapporté, il y a quelques mois, que la protéine AP 1, facteur de transcription activé par la protéine kinase $\mathrm{C}$ et ainsi probablement responsable de l'effet des esters de phoreffectués le plus souvent dans les phol sur la prolifération cellulaire, était l'équivalent cellulaire de l'oncogène de virus aviaire $v$-jun et semblait donc codé par un gène $c$-jun [ 1 , 2]. Il existe de nettes similitudes de structure entre les protéines APl/Jun et le facteur transcriptionnel GCN4 de levure [1], et de plus faibles homologies avec l'oncogène fos [3]. La protéine Fos apparaît maintenant faire partie d'un complexe protéique se fixant sur le même élément d'ADN que la protéine APl/Jun... jouant peut-être un rôle d'inhibition de la transcription alors que API est un activateur transcriptionnel. On peut faire l'hypothèse que la protéine Fos (associée à d'autres composants) et la protéine APl contrôlent finement l'expression de gènes par l'intermédiaire de leur interaction avec la même séquence d'ADN. Une expression qualitativement ou quantitativement perturbée des gènes fos et jun pourrait perturber ce contrôle et aboutir au dérèglement des mécanismes de la prolifération.

[2. Bohmann D, et al. Science 1987 ; 238: 1386-92.]

[3. Franza BR, et al. Science 1988; 239: 1150-3.]

[4. Rauscher F J, et al. Cell 1988 ; 52 : 471-80.]

Association entre le pemphigus vulgaris et des allèles du système HLA. Le pemphigus vulgaris est une maladie auto-immune due à des anticorps dirigés contre une substance localisée dans la membrane basale des kératinocytes. Une association était déjà connue avec des allèles de la classe II de la région HLA-D. Une étude menée sur des malades d'Israël et d'Autriche a montré que des polymorphismes de restriction utilisant une sonde $\mathrm{DQ} \beta$ distinguent les malades des témoins de même ethnie. Des polymorphismes situés en DQwl et DQw3 pourraient être liés au gène de susceptibilité à la maladie. L'amplification génique in vitro (PCR, polymerase chain reaction) a rendu possible l'analyse de la séquence d'un variant associé au pemphigus vulgaris [2]. L'étude de la
[1. Struhl K. Cell 1987; $50: 841-6$.] distribution de ce variant pourrait éclairer les mécanismes moléculaires sous-jacents aux associations entre maladies et gènes HLA de classe II. [1. Szafer F, et al. Proc Natl Acad Sci USA 1987 ; 84 : 6542-5.]

[2. Sinha AA, et al. Science 1988; 239: 1026-9.]

- Hérédité apparemment non mendélienne pour une sonde d'ADN. Une sonde "anonyme», appelée DXSI99, située sur le bras court du chromosome $\mathrm{X}$, a été hybridée à de l'ADN humain coupé par l'enzyme de restriction AvalI. Dans ces conditions, toutes les femmes normales ont montré deux bandes de 6,0 et $6,5 \mathrm{~kb}$ (kilobases), tous les hommes normaux une seule bande de $6,5 \mathrm{~kb}$. Le deuxième fragment de $6,0 \mathrm{~kb}$ est par contre présent chez un homme 49 XXXXY, chez lequel trois X sont inactivés. Ce variant constitue donc un marqueur du chromosome $\mathrm{X}$ inactif ; il semble dû à une différence de méthylation entre $\mathrm{X}$ actif et inactif.

[Starr T, Wood S. Am J Hum Genet 1988, 42 : 267-70.]

IGF II (insulin growth factor de type II) et le mannose 6 phosphate se lient en des sites différents de leur récepteur commun. Nous avons rapporté il y a peu de temps la détermination de la structure du récepteur commun des résidus mannose 6phosphate, servant à «cibler» les enzymes lysosomales, et du facteur de croissance IGF II [1]. L'équipe de Von Figura, de Göttingen (RFA) montre maintenant que les sites de fixation des deux ligands sont distincts, ce qui est plus aisé à concevoir qu'un site unique pour deux ligands aussi différents que le peptide IGF II et le sucre mannose 5 -phosphate. Ces deux sites de fixation correspondentils aussi à deux types différents d'activation, conduisant soit à la stimulation de la prolifération, soit à l'internalisation des enzymes lysosomales?

[1. Braulke T, et al. Biochem Biophys Res Commun 1988 ; 150 : 1287 93.]
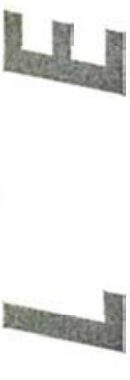

(n)

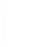
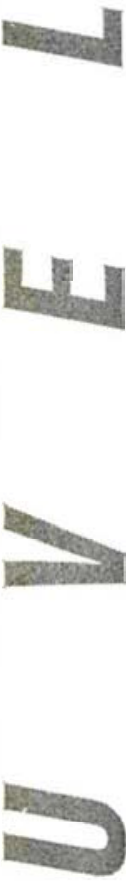

$\mathrm{m} / \mathrm{s} n^{\circ} 6$ vol. 4, juin 88 